\title{
A Chaotic Oscillator Based on HP Memristor Model
}

\author{
Guangyi Wang, Mingzhang Cui, Bozhen Cai, Xiaoyuan Wang, and Tiling Hu
}

Key Laboratory of RF Circuits and Systems, Ministry of Education of China, Institute of Modern Circuits and Intelligent Information, Hangzhou Dianzi University, Hangzhou, Zhejiang 310018, China

Correspondence should be addressed to Mingzhang Cui; cmz_666@foxmail.com

Received 15 June 2015; Revised 15 September 2015; Accepted 15 October 2015

Academic Editor: Ricardo Riaza

Copyright (C) 2015 Guangyi Wang et al. This is an open access article distributed under the Creative Commons Attribution License, which permits unrestricted use, distribution, and reproduction in any medium, provided the original work is properly cited.

\begin{abstract}
This paper proposes a simple autonomous memristor-based oscillator for generating periodic signals. Applying an external sinusoidal excitation to the autonomous system, a nonautonomous oscillator is obtained, which contains HP memristor model and four linear circuit elements. This memristor-based oscillator can generate periodic, chaotic, and hyperchaotic signals under the periodic excitation and an appropriate set of circuit parameters. It also shows that the system exhibits alternately a hidden attractor with no equilibrium and a self-excited attractor with a line equilibrium as time goes on. Furthermore, some specialties including burst chaos, irregular periodic bifurcations, and nonintermittence chaos of the circuit are found by theoretical analysis and numerical simulations. Finally, a discrete model for the HP memristor is given and the main statistical properties of this memristor-based oscillator are verified via DSP chip experiments and NIST (National Institute of Standards and Technology) tests.
\end{abstract}

\section{Introduction}

A memristor is a two-terminal circuit element, which was defined by Chua in 1971 [1]. Until 2008 the memristor with nanoscale was first realized in a $\mathrm{TiO}_{2}$ crossbar array structure form by Williams group from HP (Hewlett-Packard) labs [2]. As a new device with memory, its potential applications span different fields including nonvolatile memories on the nanoscale [3, 4], neuromorphic systems [5], chaotic circuits [6], and others $[7,8]$. Thus the HP memristor has attracted immense interest rapidly from both industry and academia [9].

In many studies, applications of memristor are one of the active topics of research, since memristor is a new nonlinear memory element and can be used in memristorbased oscillator, information encryption, memory, and so on.

The first memristor oscillator with piecewise linear (PWL) $\varphi-q$ characteristics was proposed by Itoh and Chua [10], which was obtained from Chua's circuit. Then some memristor oscillators with a PWL nonlinearity or a smooth piecewise-quadratic nonlinearity are presented based on Chua's circuit [11, 12]. Meanwhile, some other memristor oscillators based on Chua's circuit are proposed, using diverse memristor models with the $q-\varphi$ nonlinearities: $q(\varphi)=$ $a \varphi+b \varphi^{3}$ [13]. In these studies, some new properties, which are different from conventional chaos, such as equilibrium set, transient chaos, and stable chaos with an intermittence period, are found.

On the other hand, a few nonautonomous memristor oscillators are proposed. In [13], an oscillation circuit based on van der Pol circuit oscillator is implemented. Very recently, nonautonomous Chua's circuit is constructed by replacing Chua's diode with a memristor model characterized by the memductance $W(\varphi)=-a+b|\varphi(t)|$, which can exhibit some complex dynamical properties including transient chaos, transient hyperchaos, and chaotic beats [14].

Now the most common nonlinear $\varphi-q$ characteristics, including PWL, cubic, piecewise-quadratic, and absolute value function nonlinearity, of memristor are assumed and used to design memristor circuit. But $\mathrm{HP} \mathrm{TiO}_{2}$ memristor is a realized operational device, so it is of great significance for researching application circuits with the HP memristor. However, only a few studies on HP memristor-based oscillators have reported. In [6], a chaotic oscillator based on the $\mathrm{HP} \mathrm{TiO}_{2}$ memristor is introduced, which is very important for practical applications of memristor. But this circuit makes use of two HP memristors in parallel with opposite polarities, thereby showing a defect for circuit complexity. Reference [15] presents a complete model of HP memristor, which takes into consideration the interdependence between 
memristance, charge, and flux along with the boundary and initial conditions of operation. And a scroll chaotic system containing the HP memristor model and triangular wave sequence is proposed in [16], which is only a mathematical system consisting of three differential equations and contains two nonlinear functions (one of them is memristor model), showing a complexity for its mathematical structure and implemented equivalent circuit. In [11], a new chaotic system, based on the flux-controlled memductance model of the HP $\mathrm{TiO}_{2}$ memristor and Chua's circuit, is designed.

In order to generate more complex pseudorandom signals, this paper designs a nonautonomous oscillator using the mathematical model of flux-controlled memristor, which can generate periodic signals, chaotic and hyperchaotic signals, thus exhibiting more complex dynamics. In order to verify the physical realizability of the proposed oscillator, a scheme for digitally realizing this memristor oscillator is provided by using the DSP chip, and the statistical property of the chaotic oscillator is tested by using the NIST test suite. NIST test results show that randomness of the proposed oscillator is better than that of Lorenz system and is definitely up to the standards of the NIST.

The DSP technology is used to realize this memristor system, since DSP is one of mainstream techniques for information processing at present. Moreover, DSP can adapt to practical applications of both chaotic $\mathrm{PN}$ sequence generation and chaotic information encryption/decryption processing. To this end, a discrete model of HP memristor is given first.

\section{Flux-Controlled Memristance of HP Memristor}

In the seminal paper which first announced the fabrication of $\mathrm{TiO}_{2}$ memristor, the memristance is the charge-controlled form. For convenience, sometimes it is very necessary to give the flux-controlled memristance of HP memristor.

Figure 1 shows the structure of $\mathrm{HP} \mathrm{TiO}_{2}$ memristor, which is described by [2]

$$
\begin{aligned}
v(t) & =M(w) i(t) \\
& =\left[\frac{R_{\mathrm{on}} w(t)}{D}+\left(R_{\mathrm{off}}-\frac{R_{\mathrm{off}} w(t)}{D}\right)\right] i(t), \\
\frac{d w(t)}{d(t)} & =\mu_{v} \frac{R_{\mathrm{on}} i(t)}{D}, \\
M(w) & =R_{\mathrm{off}}-\frac{R_{\mathrm{off}}-R_{\mathrm{on}}}{D} w(t) \approx R_{\mathrm{off}}-\frac{R_{\mathrm{off}}}{D} w(t),
\end{aligned}
$$

where $R_{\text {off }}$ and $R_{\text {on }}$ are the high resistance and low resistance for $w=0$ and $w=D$, respectively, $\mu_{v}$ denotes the dopant mobility, $q(t)$ is the charge across the memristor, and $M(w)$ is the memristance. Other variables and parameters are shown in Figure 1.

Now, we try to find the relation between $w(t)$ and flux $\varphi(t)$ in the memristor for obtaining the flux-controlled

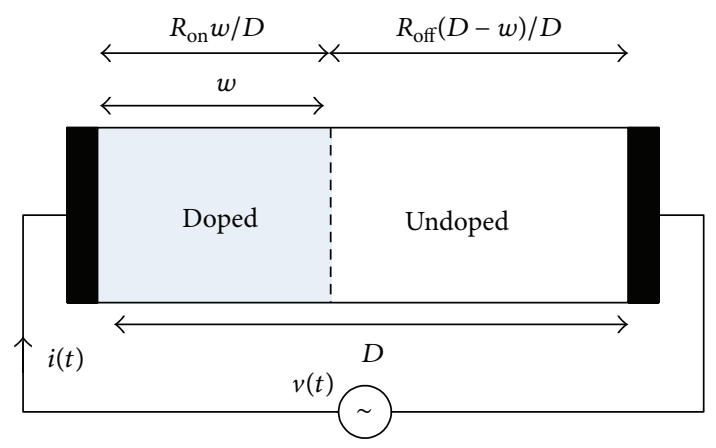

FIgURE 1: Structure of the $\mathrm{HP} \mathrm{TiO}_{2}$ memristor.

memristance. To this end we can rewrite equivalently (2) as follows:

$$
\frac{d w(t)}{d(t)}=\mu_{v} \frac{R_{\mathrm{on}}(w(t) / D) i(t)}{w(t)}=\mu_{v} \frac{v_{\mathrm{on}}}{w(t)}=\mu_{v} E_{\mathrm{on}},
$$

where $E_{\text {on }}=v_{\text {on }} / w(t)$ is the field intensity in the doped region of the memristor and $v_{\text {on }}$ is the voltage across the region. Namely, $v_{\text {on }}$ is the voltage across the resistance, $R_{\text {off }} w(t) / D$. Thus, $v_{\text {on }}$ can be written as

$$
v_{\text {on }}(t)=\frac{R_{\text {on }} w(t) / D}{R_{\text {off }}-w(t)\left(R_{\text {off }}-R_{\text {on }}\right) / D} v(t) .
$$

From (5) and (4), we can get the following equation:

$$
\frac{d w(t)}{d(t)}=\frac{\mu_{v} R_{\mathrm{on}} v(t) / D}{R_{\mathrm{off}}-w(t)\left(R_{\mathrm{off}}-R_{\mathrm{on}}\right) / D} .
$$

By integrating (6) with respect to time $t$, the relation between $w(t)$ and $\varphi(t)$ is described as

$$
\frac{1}{2}\left(R_{\text {on }}-R_{\text {off }}\right) w(t)^{2}+D R_{\text {off }} w(t)-\mu_{v} R_{\text {on }} \varphi(t)=0,
$$

where $\varphi(t)=\int_{t_{0}}^{t} v(\tau) d \tau$. By solving (7), the relationship between $w(t)$ and flux $\varphi(t)$ can be obtained as follows:

$$
w(t)=\frac{-D R_{\text {off }} \pm \sqrt{D^{2} R_{\text {off }}^{2}+2\left(R_{\text {on }}-R_{\text {off }}\right) \mu_{v} R_{\text {on }} \varphi(t)}}{R_{\text {on }}-R_{\text {off }}} .
$$

By inserting (8) into (3) the flux-controlled memristance of this memristor is obtained, which is simplified to

$$
M(\varphi)=k_{1} \pm\left(k_{2}+k_{3} \varphi\right)^{1 / 2}
$$

where $k_{1}=-R_{\text {on }} R_{\text {off }} /\left(R_{\text {off }}-R_{\text {on }}\right), k_{2}=R_{\text {off }}^{4} /\left(R_{\text {off }}-R_{\text {on }}\right)^{2}$, and $k_{3}=2 R_{\text {on }} R_{\text {off }}^{2} \mu_{v} / D^{2}\left(R_{\text {off }}-R_{\text {on }}\right)$.

In (9), because $R_{\text {off }} \gg R_{\text {on }}, k_{1}$ is negative and $k_{2}$ and $k_{3}$ are all positive; therefore $M(\varphi)$ has both positive and negative values, corresponding to both passive and active memristors, respectively. 


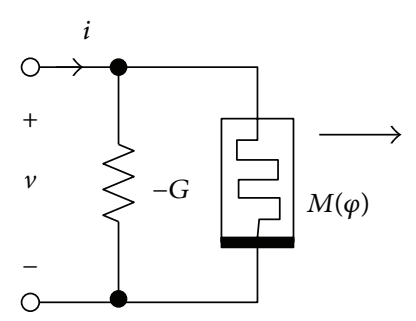

(a)

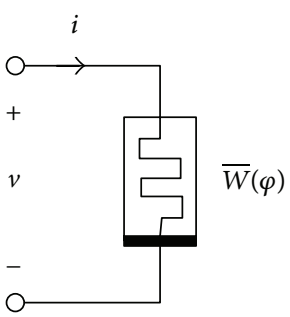

(b)

FIgURE 2: Equivalent active memristor with a passive HP memristor. (a) Two-terminal circuit. (b) Equivalent active memristor.

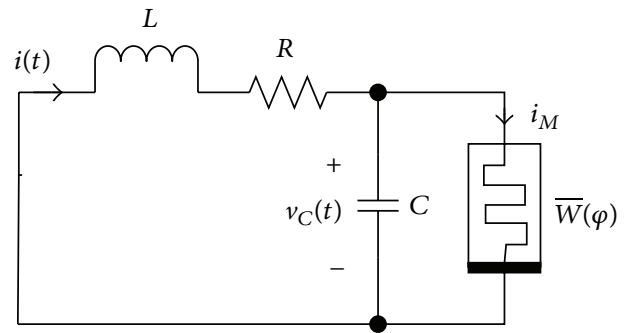

(a)

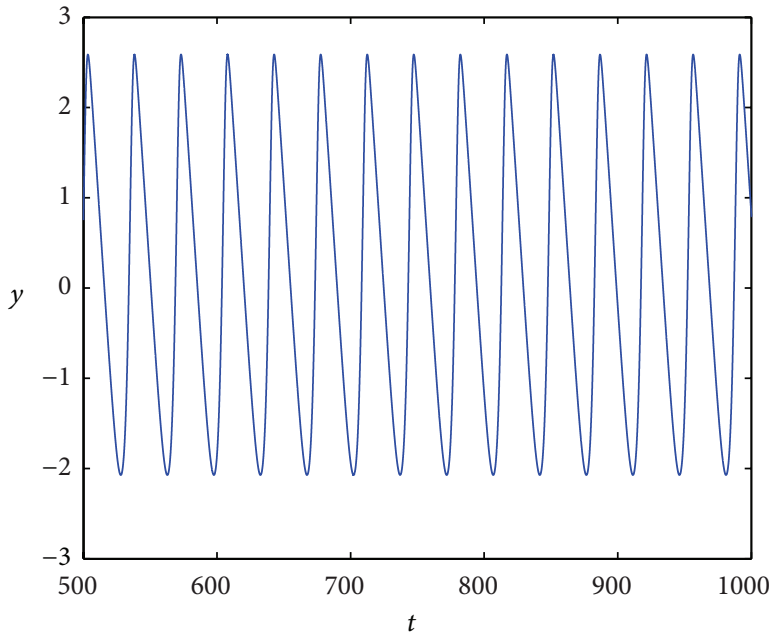

(b)

Figure 3: Autonomous flux-controlled memristor system. (a) Circuit. (b) Periodic oscillation waves.

By using the HP memristor model, we can design simplest autonomous oscillator for generating periodic or chaotic oscillations.

Figure 2 shows a two-terminal circuit, which consists of a passive HP memristor and a negative conductance (or a negative resistance). The equivalent memductance of this two-terminal circuit is given by

$$
\bar{W}(\varphi)=\frac{1}{M(\varphi)}-G,
$$

where $M(\varphi)=k_{1}+\left(k_{2}+k_{3} \varphi\right)^{1 / 2}>0$. When $G>1 / M(\varphi)$, this two-terminal circuit becomes an active memristive system; that is, $\bar{W}(\varphi)=1 / M(\varphi)-G<0$, implying that the circuit can be regarded as an equivalent active memductance (or an equivalent active memristance) shown in Figure 2(b).

\section{An Autonomous Memristor Oscillator}

A simple autonomous memristor system is designed as in Figure 3(a), which consists of a resistor $R$, an inductor $L$, a capacitor $C$, a negative resistor $-G$, and active flux-controlled memductance $\bar{W}$.

By applying Kirchoff's laws to this system, the state equations for the voltage $v_{C}$ across the capacitor $C$, the current $i$ through the inductor $L$ and the flux $\varphi$ in the active memductance $\bar{W}$ are represented by the following differential equations:

$$
\begin{aligned}
\frac{C d v_{C}}{d t} & =i-\bar{W}(\varphi) v_{C} \\
\frac{L d i}{d t} & =-i R-v_{C} \\
\frac{d \varphi}{d t} & =v_{C}
\end{aligned}
$$

where $\bar{W}(\varphi)=1 / M(\varphi)-G=1 /\left(k_{1}+\left(k_{2}+k_{3} \varphi\right)^{1 / 2}\right)-G$. Rescaling (11) as $t=R C \tau, v_{C}=x, R i=y, \varphi=R C z$, and $\beta=R^{2} C / L$ and then redefining $\tau$ as $t$, the following set of normalized equations are obtained:

$$
\begin{aligned}
& \dot{x}=y-\bar{W}(z) x, \\
& \dot{y}=-\beta(x+y), \\
& \dot{z}=x,
\end{aligned}
$$

where $\bar{W}(z)=R /\left(k_{1}+\left(k_{2}+k_{3}^{\prime} z\right)^{1 / 2}\right)-R G$ and $k_{3}^{\prime}=R C k_{3}$. If we take the values of the flux-controlled memristor parameters, $R_{\text {on }}=0.17 \mathrm{k} \Omega, R_{\text {off }}=1.2 \mathrm{k} \Omega, \mu_{v}=10^{-10} \mathrm{~cm}^{2} \mathrm{~s}^{-1} \mathrm{v}^{-1}$, and $D=3.5 \mathrm{~nm}$, the corresponding parameters are $k_{1}=-0.20$, $k_{2}=1.96$, and $k_{3}^{\prime}=0.039$. 


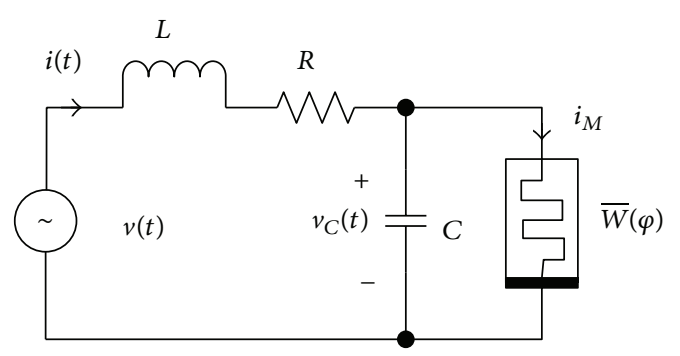

FIGURE 4: Nonautonomous memristor circuit.

When $R=1 \mathrm{k} \Omega, G=0.891 \mathrm{mS}, L=2 \mathrm{H}$, and $C=$ $100 \mathrm{nF}$, the parameter $\beta$ is 0.05 . Simulation results show that this autonomous system with these parameters is a periodic oscillator, whose waves are shown in Figure 3(b).

\section{A Nonautonomous Memristor Oscillator and Its Dynamics}

4.1. A Nonautonomous Memristor Oscillator. Applying an external sinusoidal excitation voltage to the autonomous system, a nonautonomous system is obtained, which is shown in Figure 4, where $v(t)=f \sin (\Omega t)$. Then (11) and (12) can be translated into (13) and (14), respectively; those are

$$
\begin{aligned}
\frac{C d v_{C}}{d t} & =i-\bar{W}(\varphi) v_{C}, \\
\frac{L d i}{d t} & =-i R-v_{C}+f \sin \Omega t, \\
\frac{d \varphi}{d t} & =v_{C}, \\
\dot{x} & =y-\bar{W}(z) x, \\
\dot{y} & =-\beta(x+y)+F \sin \omega t, \\
\dot{z} & =x,
\end{aligned}
$$

where $F=\beta f$ and $\omega=\Omega R C$.

If we, respectively, change the inductor parameters $L$ and $G$ from $2 \mathrm{H}$ and $0.891 \mathrm{mS}$ to $2.65 \mathrm{H}$ and $0.791 \mathrm{mS}$ while maintaining all other circuit parameters unchanged, taking $\Omega=850 \mathrm{~Hz}$ and $f=14.15 \mathrm{~V}$, the corresponding parameters of system (14) are $\beta=0.378, F=5.35, \omega=0.085, k_{1}=-0.2$, $k_{2}=2.08$, and $k_{3}^{\prime}=0.039$. Under the conditions of these parameters, this system exhibits a chaotic attractor, as shown in Figure 5.

Notice that Figure $5(\mathrm{~d})$ shows the chaotic $v_{C}-i_{M}$ hysteresis curves of the flux-controlled memristor, which contains a lot of nonrepeating $v_{C}-i_{M}$ curves, thereby demonstrating chaotic properties of this system.

4.2. Basic Dynamical Characteristics. From system (14), one has

$$
\nabla V=\frac{\partial \dot{x}}{\partial x}+\frac{\partial \dot{y}}{\partial y}+\frac{\partial \dot{z}}{\partial z}+\frac{\partial \dot{w}}{\partial w}=R\left(G-\frac{1}{M(z)}\right)-\beta .
$$

Therefore, to make system (14) dissipative, it must satisfy

$$
\frac{1}{[1 / M(z)-G]}<\frac{R}{\beta}
$$

that is, in order to generate chaotic attractors, the total resistance for parallel connection of the negative resistor and the memristor in Figure 3 must be less than the value of one $\beta$ of the linear resistor $R$. To this end, the system must meet $z<122.6$ for the above fixed system parameters.

Strictly speaking, the equilibria of system (14) are related with excitation voltage. When $\sin \Omega t \neq 0$, that is, $\Omega t \neq$ $k \pi(k=0,1,2, \ldots)$, this system is written as

$$
\begin{aligned}
& \dot{x}=y-R \bar{W}(z) x, \\
& \dot{y}=-\beta(x+y)+v, \\
& \dot{z}=x,
\end{aligned}
$$

where $v \neq 0$ and $v$ is an instantaneous value of the excitation voltage at time $t(t \neq k \pi / \Omega)$.

Let $\dot{x}=0, \dot{y}=0$ and $\dot{z}=0$; (17) has no solution of equilibria, and then it becomes a system with no equilibria, which is possible to display hidden attractors without any equilibria [9].

When $\sin \Omega t=0$, that is, $\Omega t=k \pi(k=0,1,2, \ldots)$, or the external excitation equals zero, that is, $\sin \Omega t \equiv 0$, (14) becomes a three-dimensional autonomous system, which corresponds to (12). At the moment, it has an equilibrium set, which is obtained by solving the equations $\dot{x}=\dot{y}=\dot{z}=0$ :

$$
S=\{(x, y, z) \mid x=y=0, z=c\},
$$

where $c$ is a real arbitrary constant, thereby implying that these equilibria occupy the whole $z$-axis, which is also called a line equilibrium [9].

Considering the above two cases together, that is, $\Omega t \neq k \pi$ and $\Omega t=k \pi(k=0,1,2, \ldots)$, we can see that the system switches alternately between a nonautonomous subsystem with no equilibrium and an autonomous subsystem with a line equilibrium under the control of sine voltage excitation. Therefore a hidden attractor and a self-excited attractor appear exchangeably with the time evolution.

Particularly, when the external sine excitation is removed from the nonautonomous system, that is, $\sin \Omega t \equiv 0$, it will become the autonomous system shown in Figure 3.

For the two cases of $\sin \Omega t \equiv 0$ and $\Omega t=k \pi(k=$ $0,1,2, \ldots)$, both of them have the same Jacobian matrix at the same equilibrium set $S$ :

$$
J=\left[\begin{array}{ccc}
G-\frac{1}{M(w)} & 1 & 0 \\
-\beta & -\beta & 0 \\
1 & 0 & 0
\end{array}\right]
$$

And the characteristic equation is derived as

$$
\lambda\left(\lambda^{2}+a_{1} \lambda+a_{2}\right)=0
$$




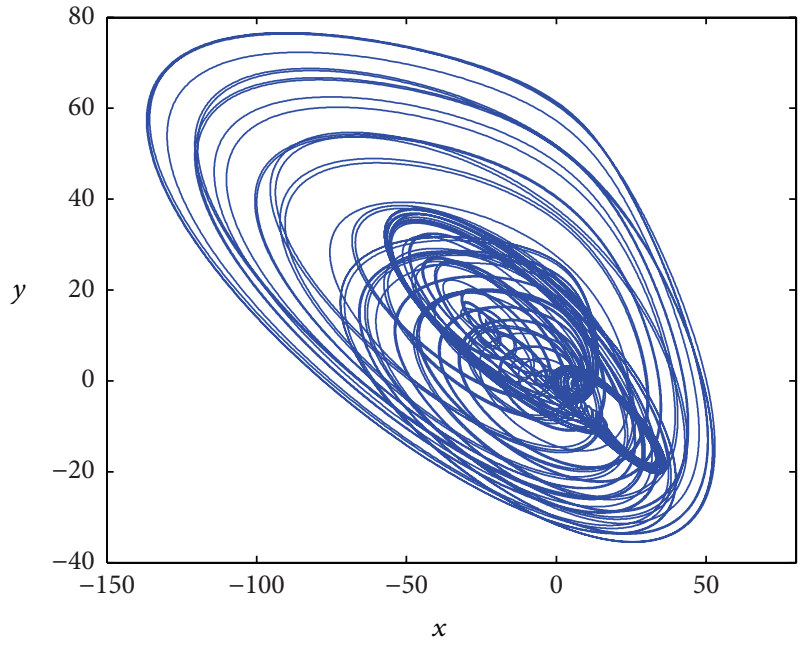

(a)

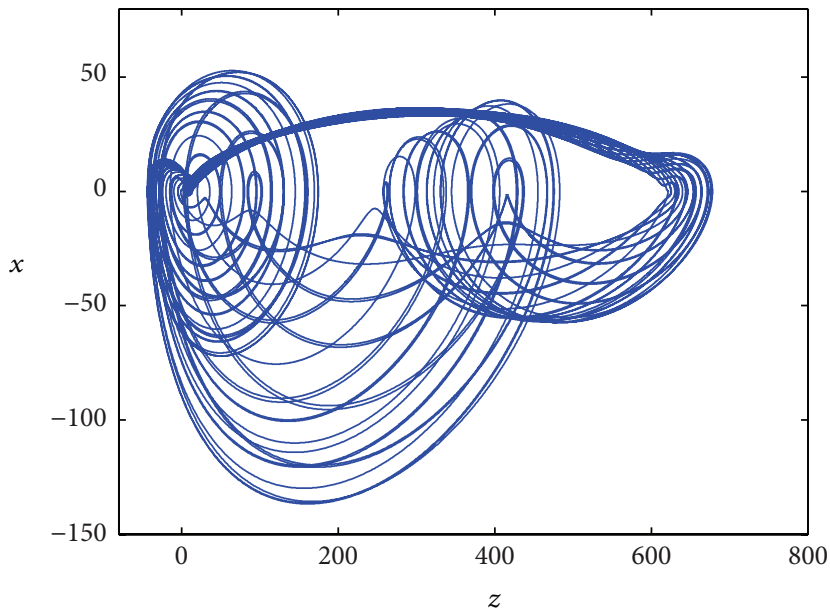

(c)

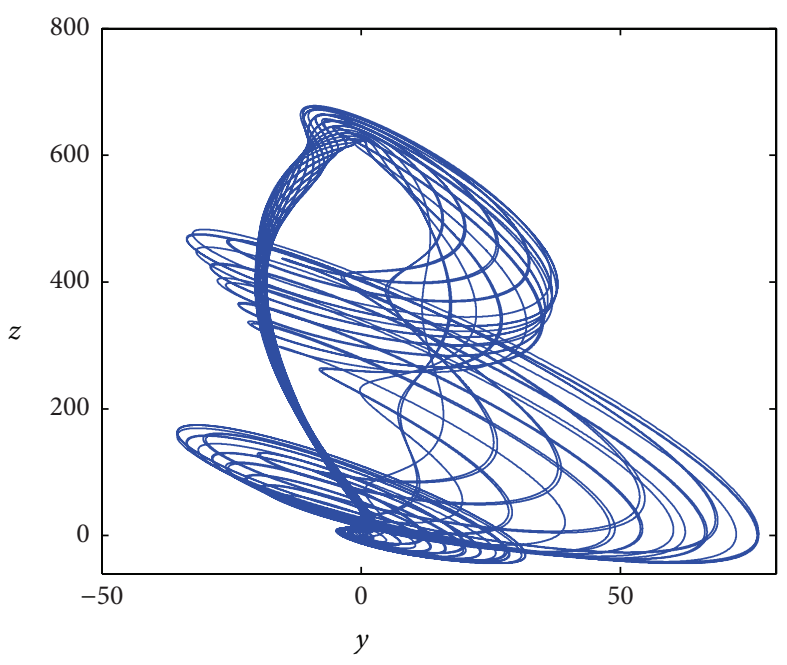

(b)

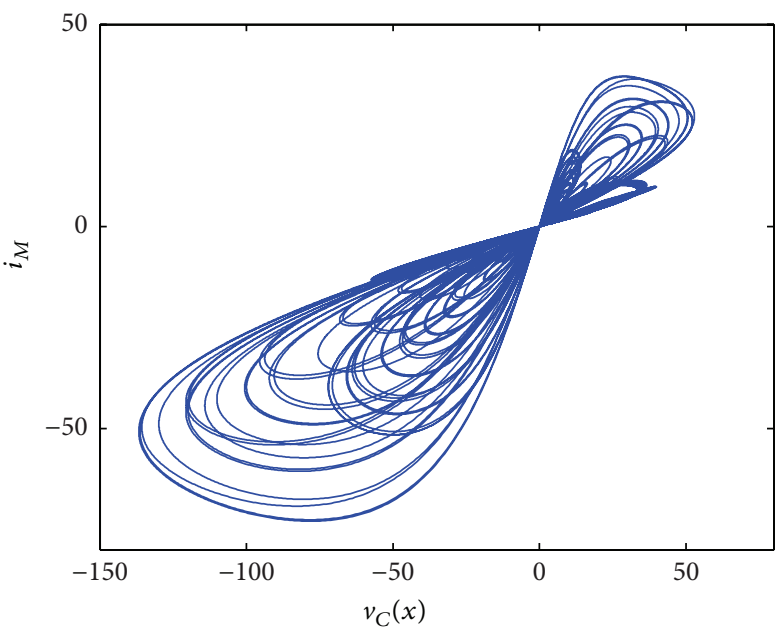

(d)

FIgURE 5: Chaotic attractors of system (13). (a) $v_{C}-i$ plane. (b) $i-\varphi$ plane. (c) $\varphi-v_{C}$ plane. (d) $v_{C}-i_{M}$ plane.

where $a_{1}=\beta-(G-1 / M(w))$ and $a_{2}=\beta-\beta(G-1 / M(w))$. By solving (20), we obtain one eigenvalue, that is, $\lambda_{1}=0$, and one quadratic equation of $\lambda$ :

$$
\lambda^{2}+a_{1} \lambda+a_{2}=0 .
$$

According to Routh-Hurwitz condition, all rotes of (21) have negative real components if and only if $a_{1}>0$ and $a_{1} a_{2}>$ 0 ; that is, $\beta-(G-1 / M(\varphi))>0$ and $\beta-\beta(G-1 / M(\varphi))>0$. Thus one has

$$
\begin{aligned}
& \varphi<\frac{1}{k_{3}}\left(\frac{1}{G-\beta}-k_{1}\right)^{2}-\frac{k_{2}}{k_{3}}, \\
& \varphi<\frac{1}{k_{3}}\left(\frac{1}{G-1}-k_{1}\right)^{2}-\frac{k_{2}}{k_{3}} .
\end{aligned}
$$

For the above typical parameters of generating chaos, system (11) is unstable when

$$
\varphi>123.64 \mathrm{mWb}
$$

thereby the dynamics of the memristor system without the external excitation is determined by the initial values of flux $\varphi$ of the memristor.

On the other hand, by solving (20), we obtain the eigenvalues of Jacobian matrix (19):

$$
\begin{aligned}
& \lambda_{1}=0 \\
& \lambda_{23}= \begin{cases}\frac{-a_{1} \pm\left(a_{1}^{2}-4 a_{2}\right)^{1 / 2}}{2} & \left(a_{1}^{2}-4 a_{2} \geq 0\right) \\
\frac{-a_{1} \pm\left(4 a_{2}-a_{1}^{2}\right)^{1 / 2} j}{2} & \left(a_{1}^{2}-4 a_{2}<0\right) .\end{cases}
\end{aligned}
$$

Figure 6 shows the eigenvalues with respect to the equilibrium $c$, which can determine stability of the system. When $c<-52, S$ is an unstable saddle point with a positive real root, a negative real root, and a zero root. With the increasing of $c$, absolutes of the two real roots increase quickly and reach a maximum at $c=52$ and then become two complex conjugate roots with the same negative real components when $c \geq-52$. 


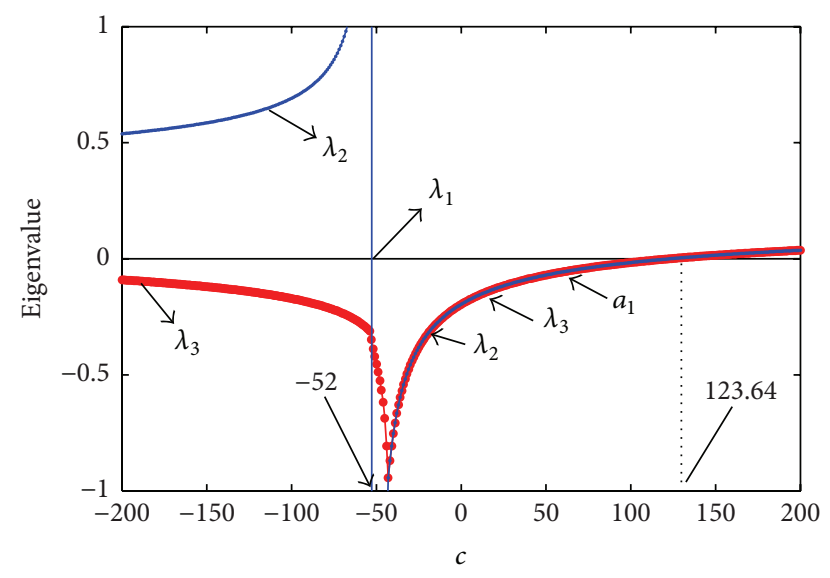

FIGURE 6: Eigenvalues of (20) varying with $c$.

At $c=123.64$, the two real components become positive, yielding unstable focus.

4.3. Chaos and Hyperchaos in Bifurcations. The dynamics of (14) depend on the parameters $F, \omega$, and $\beta$ and other circuit parameters. Now we begin to explore the dynamical characteristics varying with these parameters. To start with, we observe the bifurcation diagram and Lyapunov exponent spectrum with respect to $F$, as shown in Figure 7, where $\beta=$ $0.378, \omega=0.085, k_{1}=-0.2, k_{2}=2.08$, and $k_{3}=0.039$. By increasing the amplitude $F$ from zero, the system exhibits the bifurcation route from periodic orbits without positive exponent to chaos with one positive exponent, followed by hyperchaos with two positive exponents. In particular, a novel phenomenon occurs at $F=4.5$, where a period 3 orbit and an irregular period bifurcation appear but without obvious period-doubling bifurcations. Figure 8 shows the corresponding hyperchaotic, chaotic, and periodic orbits.

Figure 9 shows the bifurcation diagram and the corresponding Lyapunov exponent spectrum with respect to $\omega$, in which burst chaos occurs at $\omega=0.082$ and the system undergoes complex bifurcations from chaos to complex periodic orbits to chaos, where irregular period bifurcation occurs again.

Figure 10 shows the bifurcation diagram and corresponding Lyapunov exponent spectrum for $x$ with respect to the parameter $\beta$ related with the circuit parameters $R, L$, and $C$. From Figure 8 we can see that with the increase of $\beta$, the system also exhibits a complex evolution law from period to chaos to hyperchaos, and it eventually evolves to a periodic orbit.

\section{Oscillator with a Nonlinear Drift Model of Memristor}

The memristor model described in (1)-(4) is a linear drift model. However, according to the experimental observation in the HP memristor, the drift speed $d w / d t$ is nonlinear for the current $i(t)$ through the memristor. So we describe

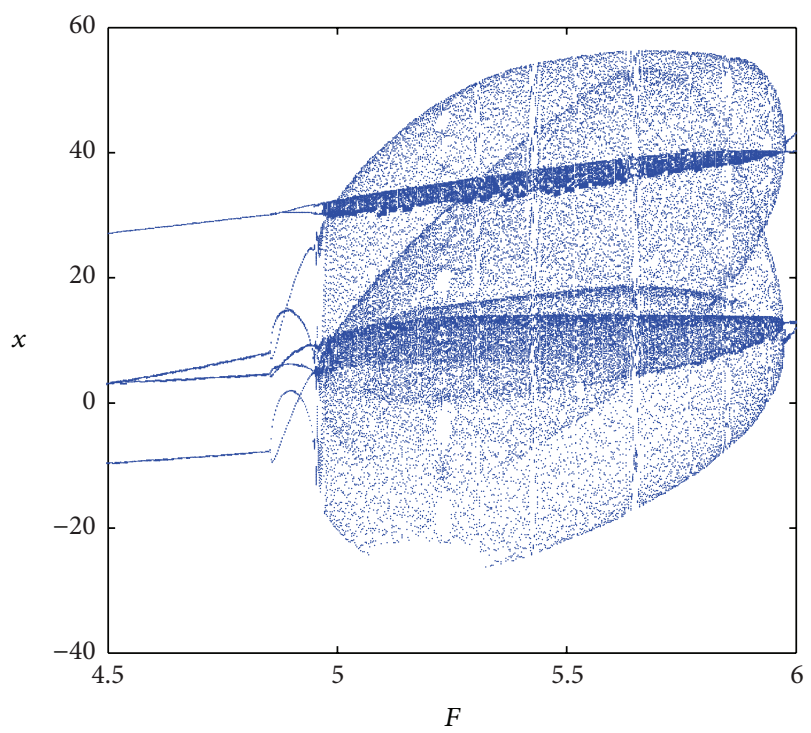

(a)

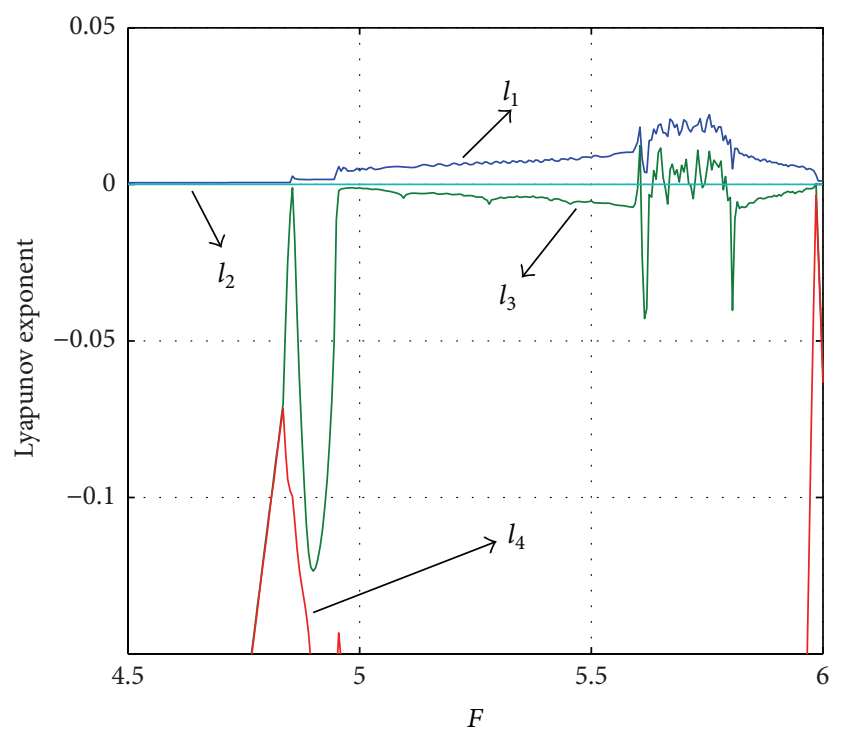

(b)

FIGURE 7: Bifurcation diagram and Lyapunov exponent spectra with respect to $F$. (a) Bifurcation diagram; (b) Lyapunov exponent spectrum.

the real memristor by using the following nonlinear drift model [6]:

$$
\begin{aligned}
M(w) & =R_{\mathrm{off}}-\frac{R_{\mathrm{off}}-R_{\mathrm{on}}}{D} w(t), \\
\frac{d w(t)}{d t} & =\frac{\mu_{v} R_{\mathrm{on}}}{D} F\left(\frac{w(t)}{D}\right) i(t), \\
F_{J}(x, i) & =1-(x-\operatorname{stp}(i))^{2},
\end{aligned}
$$

where $x=w / D ; \operatorname{stp}(i)=0$ for $i<0$ and $\operatorname{stp}(i)=1$ for $i>0$ or $i=0$. 


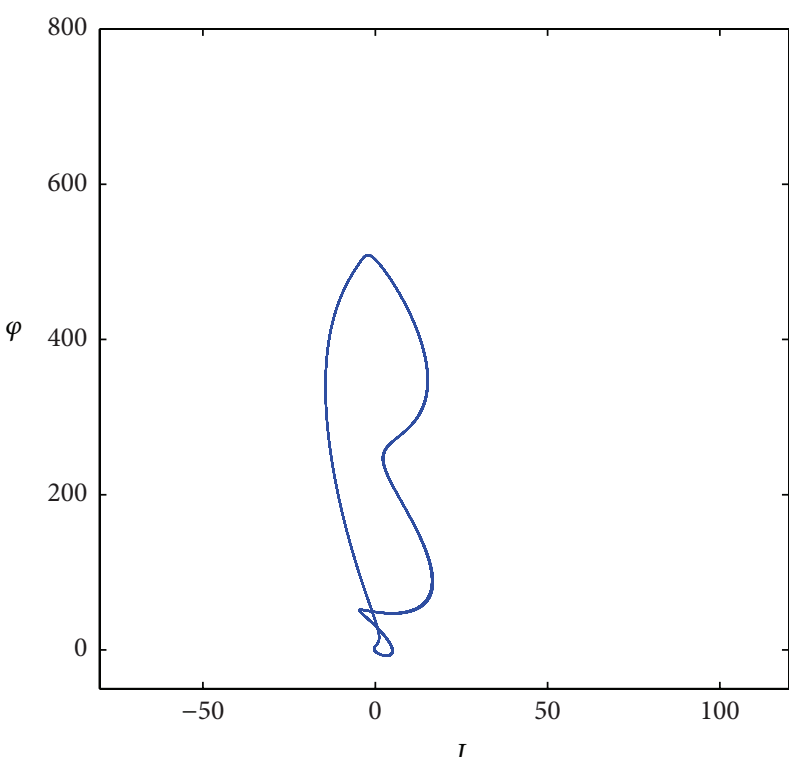

(a)

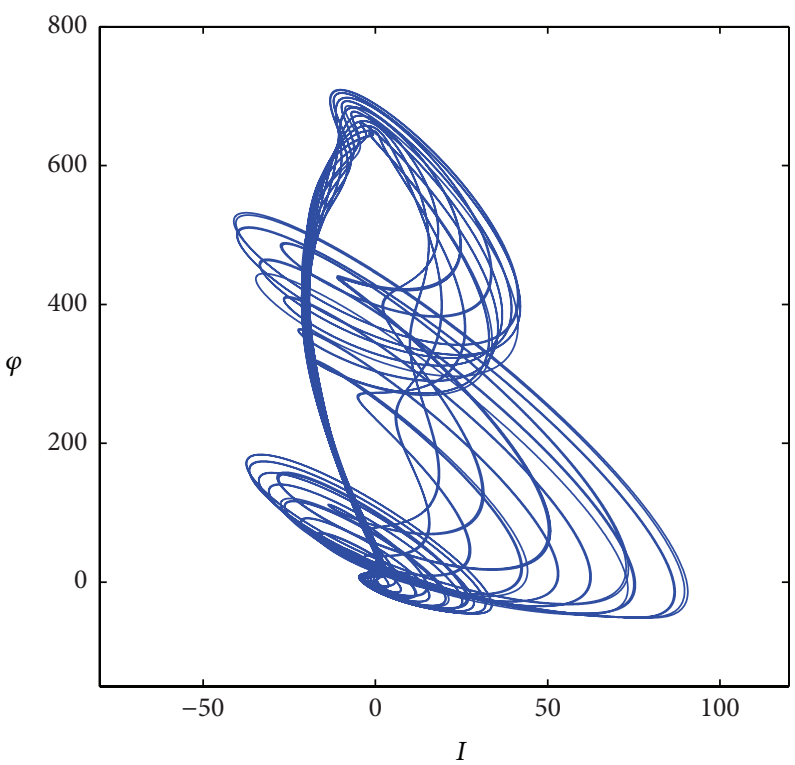

(c)

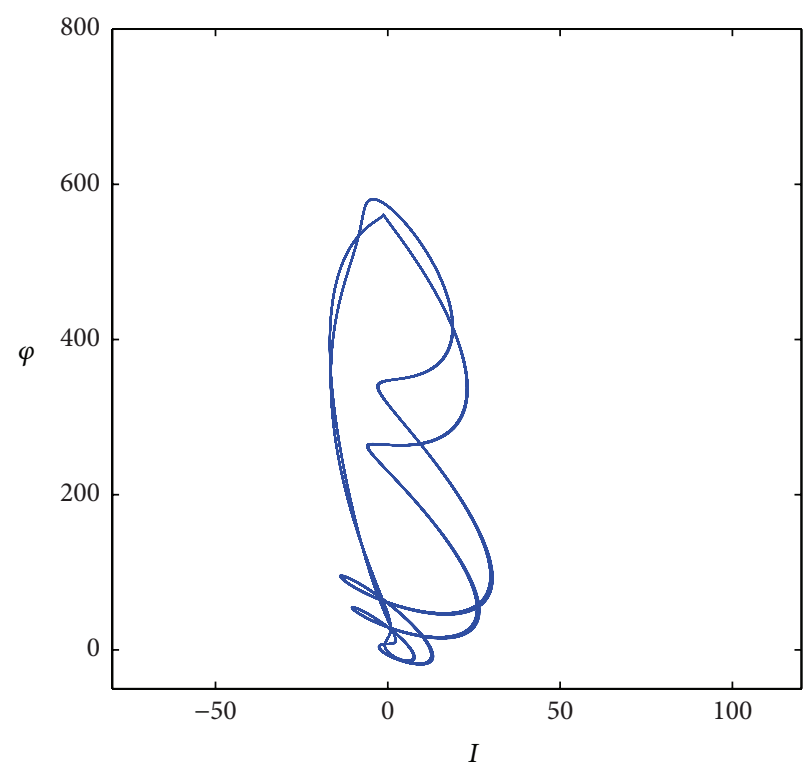

(b)

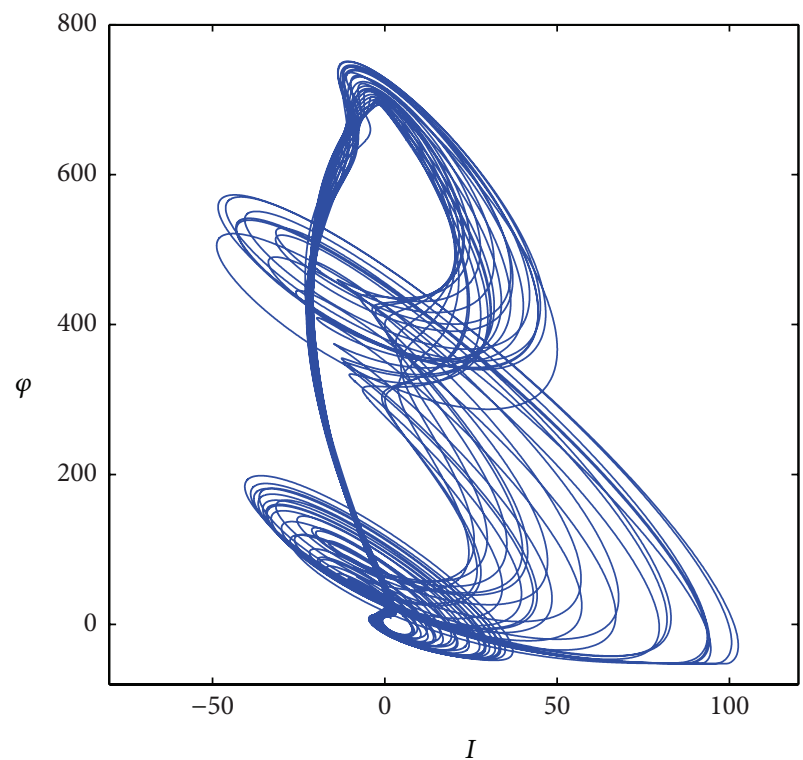

(d)

Figure 8: Evolutions with $F$ from period to chaos to hyperchaos. (a) Period with $F=4.5$. (b) Period with $F=4.9$. (c) Chaos with $F=5.5$. (d) Hyperchaos with $F=5.75$.

If we take the nonlinear drift model of (25), the state equations of the circuit shown in Figure 4 are described by

$$
\begin{aligned}
\frac{C d v_{C}}{d t} & =i+G v_{C}-\frac{v_{C}}{M(w)}, \\
\frac{L d i}{d t} & =-i R-v_{C}+f \sin \Omega t, \\
\frac{d w}{d t} & =\frac{\mu_{v} R_{\text {on }} F_{J}(w / D) v_{C}}{(D M(w))}, \\
M(w) & =R_{\text {off }}-\frac{\left(R_{\text {off }}-R_{\text {on }}\right) w}{D} .
\end{aligned}
$$

Let $t=R C \tau, v_{C}=x, R i=y, w=R C z$, and $\beta=R^{2} C / L ;(26)$ is written as

$$
\begin{aligned}
\frac{d x}{d t} & =y+G x-\frac{x}{M(z)}, \\
\frac{d y}{d t} & =-\beta(y+x)+F \sin \omega t, \\
\frac{d z}{d t} & =\frac{\mu_{v} R_{\mathrm{on}} F_{J}(z / D) x}{(D M(z))}, \\
M(z) & =R_{\mathrm{off}}-\frac{\left(R_{\mathrm{off}}-R_{\mathrm{on}}\right) z}{D} .
\end{aligned}
$$




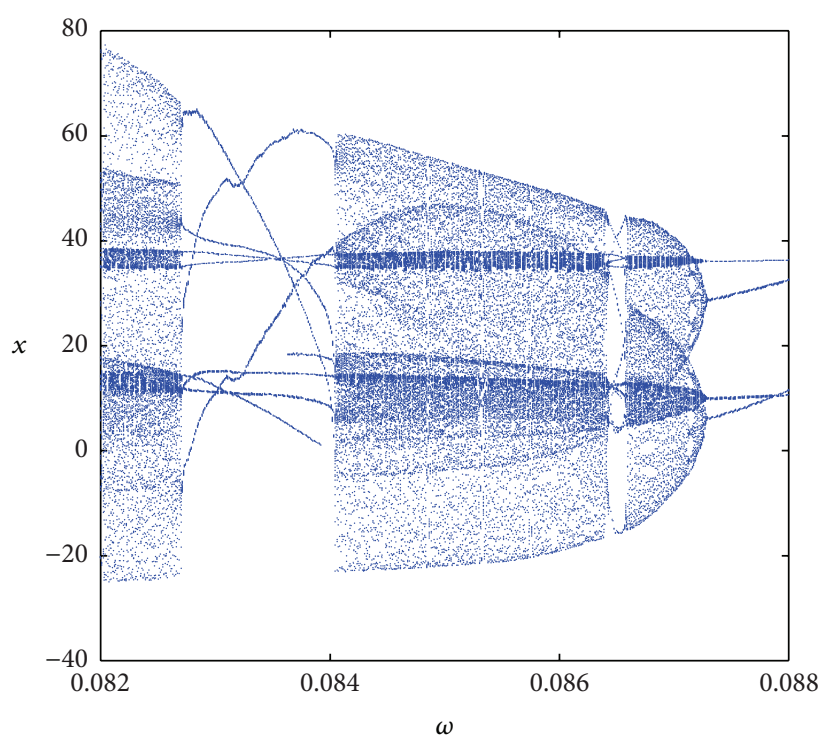

(a)

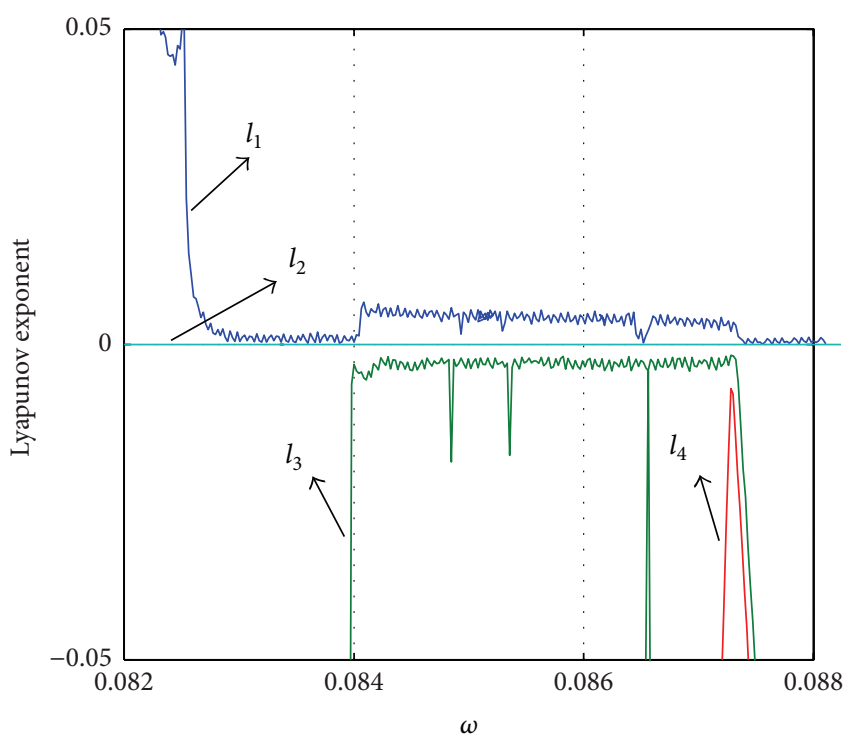

(b)

FIGURE 9: Bifurcation diagram and the corresponding Lyapunov exponent spectrum with respect to $\omega$. (a) Bifurcation diagram. (b) Lyapunov exponent spectrum.

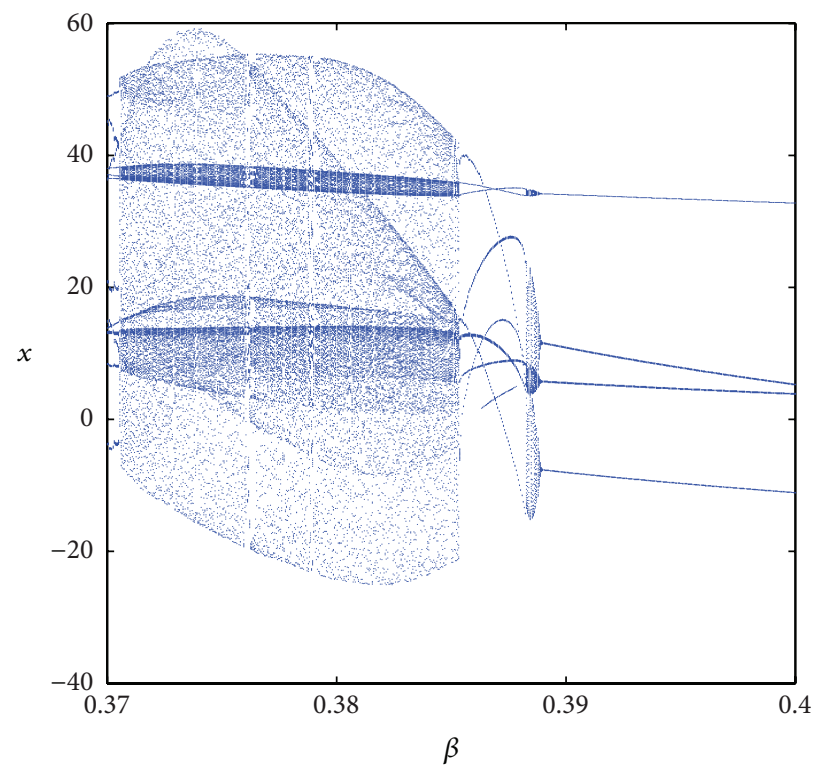

(a)

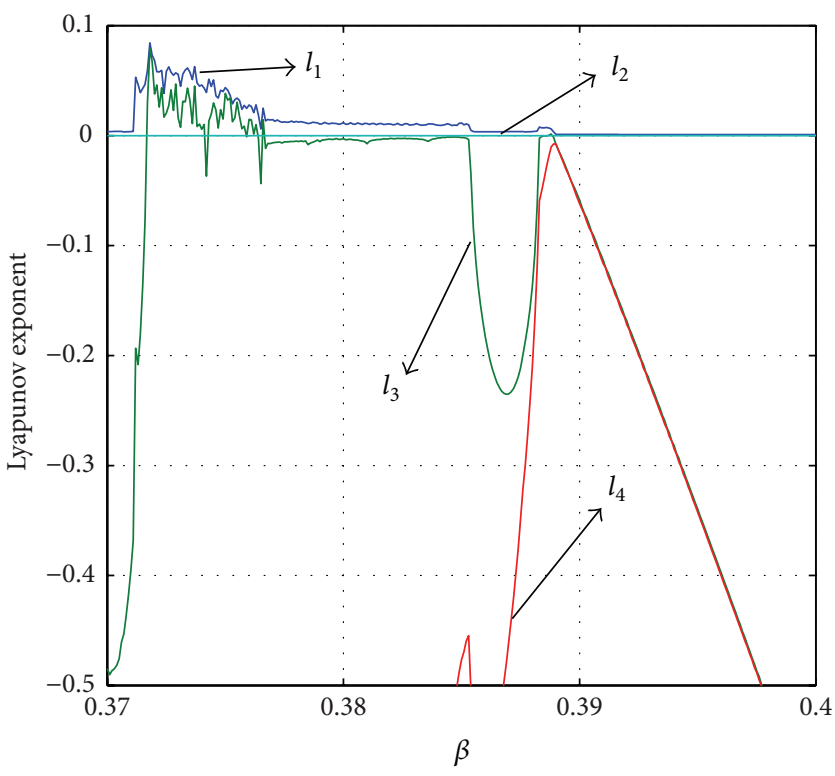

(b)

FIGURE 10: Bifurcation diagram and the corresponding Lyapunov exponent spectrum with respect to $\beta$.

When $\beta=0.205, F=0.75, \omega=1.5, G=0.2 \mathrm{mS}$, $R_{\text {on }}=0.9 \mathrm{k} \Omega, R_{\text {off }}=2.5 \mathrm{k} \Omega, D=3.0 \mathrm{~nm}$, and $\mu_{v}=$ $10^{-14} \mathrm{~cm}^{2} \mathrm{~s}^{-1} \mathrm{v}^{-1}$, this oscillator with a nonlinear drift model produces a chaotic oscillation and its attractors are shown in Figure 11, where the Lyapunov exponents of this oscillator are $l_{1}=0.115, l_{2}=0$, and $l_{3}=-0.114$.

\section{Digital Realization of the Memristor System}

Now, we provide a scheme for digitally realizing the proposed flux-controlled memristor system.
The DSP technology is used to realize this memristor system, since DSP is one of mainstream techniques for information processing at present. Moreover, DSP can adapt to practical applications of both chaotic $\mathrm{PN}$ sequence generation and chaotic information encryption/decryption processing. To this end, a discrete model of HP memristor is given first.

According to (1)-(3), the discrete model of memristance can be obtained:

$$
M\left(q_{n}\right)=R_{\mathrm{off}}-\frac{\left(R_{\mathrm{off}}-R_{\mathrm{on}}\right) \mu_{v} R_{\mathrm{on}}}{D^{2}} \sum_{j=-\infty}^{n} i\left(\varsigma_{j}\right) \Delta t_{j},
$$




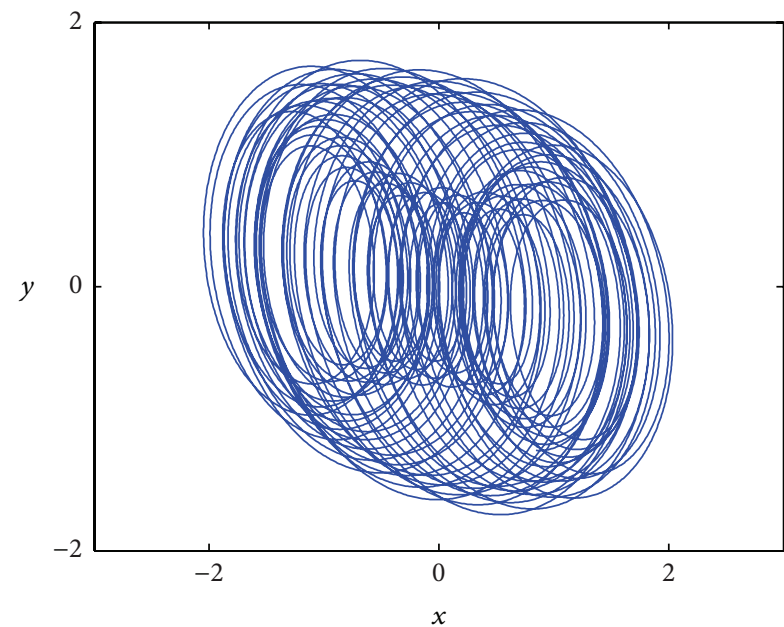

(a)

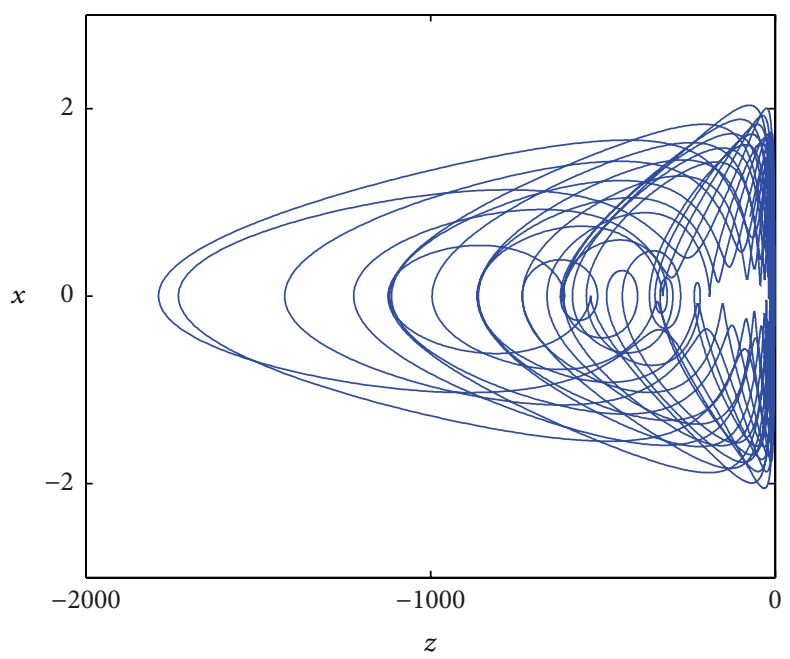

(c)

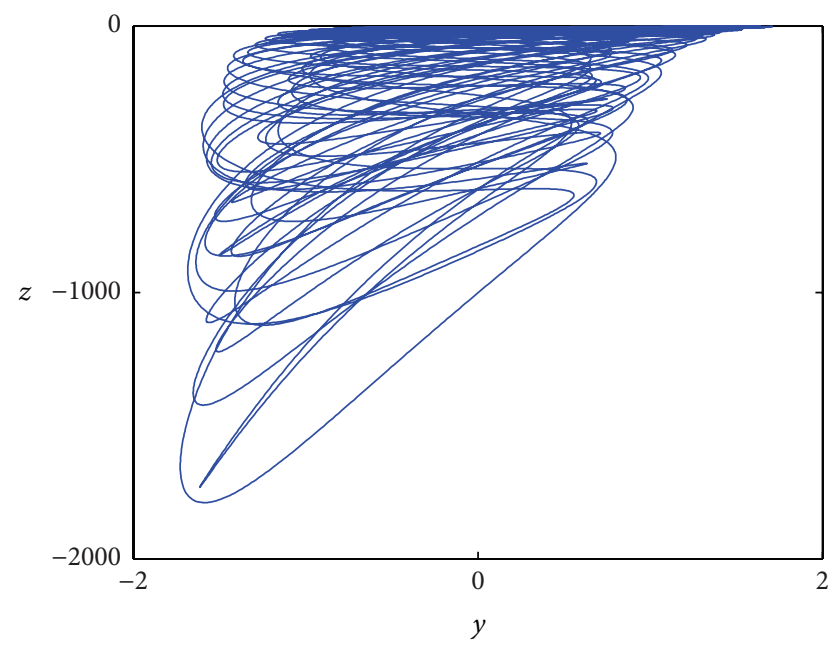

(b)

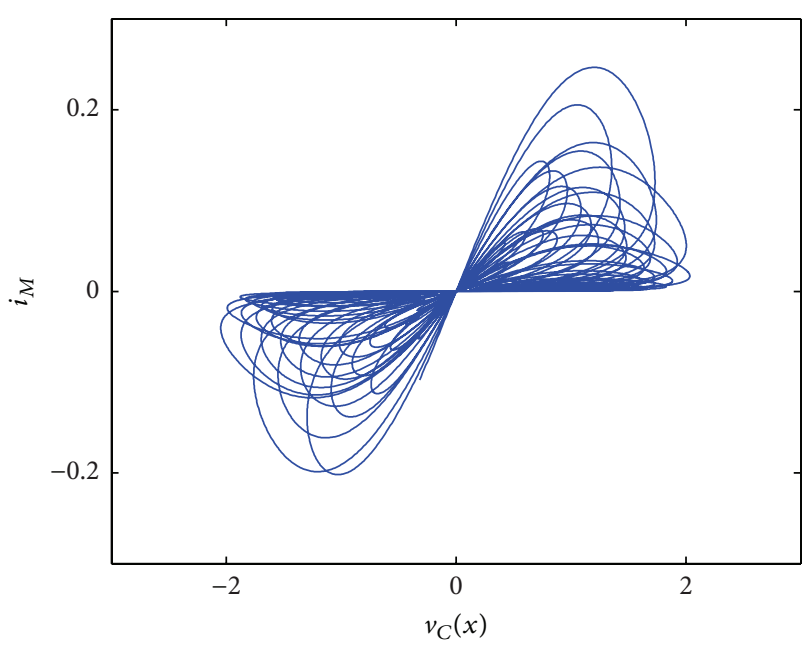

(d)

Figure 11: Chaotic attractors of system (27). (a) $x-y$ plane. (b) $y-z$ plane. (c) $z-x$ plane. (d) $x\left(v_{C}\right)-i_{M}$ plane.

where $\sum_{j=-\infty}^{n} i\left(\varsigma_{j}\right) \Delta t_{j}=q_{n}$. Hence, from (28) and (3)-(8) the discrete model of the flux-controlled memristor is derived:

$$
M\left(\varphi_{n}\right)=k_{1}+\left(k_{2}+k_{3} \varphi_{n}\right)^{1 / 2} .
$$

By applying Euler's method to (14), the discrete equations of the memristor system are described as

$$
\begin{aligned}
& x_{n+1}=x_{n}+\tau y_{n}+\tau\left(R G-\frac{R}{M\left(z_{n}\right)}\right) x_{n}, \\
& y_{n+1}=y_{n}-\tau \beta\left(x_{n}+y_{n}\right)+\tau F \sin \omega t_{n}, \\
& z_{n+1}=z_{n}+\tau x_{n} .
\end{aligned}
$$

Based on the DSP evaluation board (ICETEK-VC5509AE-sampling frequency is $5 \mathrm{M} / \mathrm{S}$ and 8 bits' quantization) and the software platform (CCStudio_v3.3), the flux-controlled memristor system is experimentally realized. Figure 12 shows the experimental orbits of period and chaos, observed by an analog oscilloscope, and the hyperchaotic orbits are shown in Figure 13. Compared with Figures 5 and 8, these experimental orbits are in good agreement with the Matlab simulation results.

Based on the DSP technology, digital pseudorandom sequences, or digital chaotic sequences, can be obtained by using the proposed memristor oscillator, which can be used in various applications of information safety. Figure 14 shows the digital pseudorandom sequence generated by experimental DSP evaluation board and observed by a digital oscilloscope, in which upper wave is the clock pulses and the lower wave is the chaotic pseudorandom sequences.

Now, we evaluate the randomness of the proposed memristor oscillator by testing its binary sequences and using the NIST test suite and compare the randomness with that of the well-known Lorenz system.

The NIST test suite is the most authoritative tool for pseudorandom test currently, which contains 15 tests. The 2.0 version of the test suite package is used in this paper. 


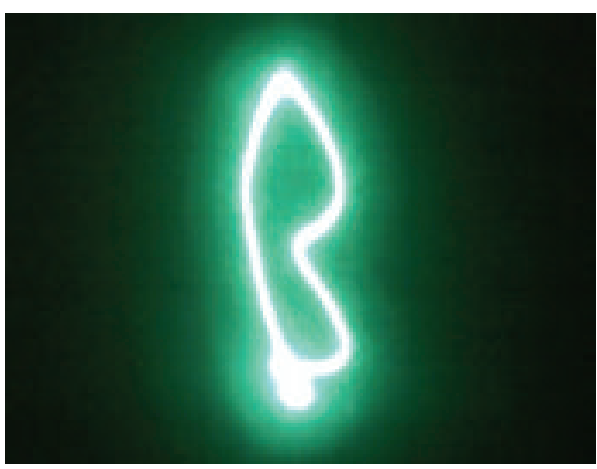

(a)

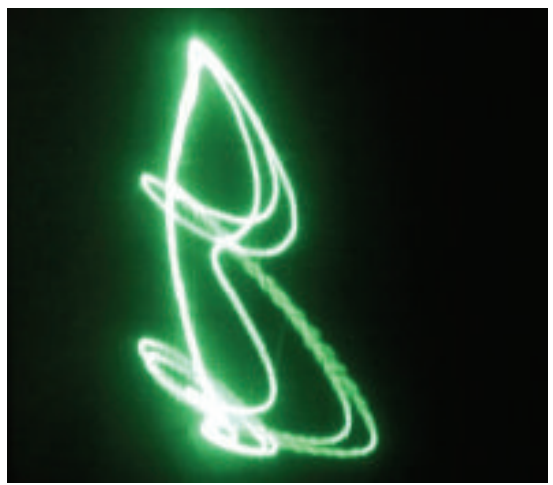

(c)

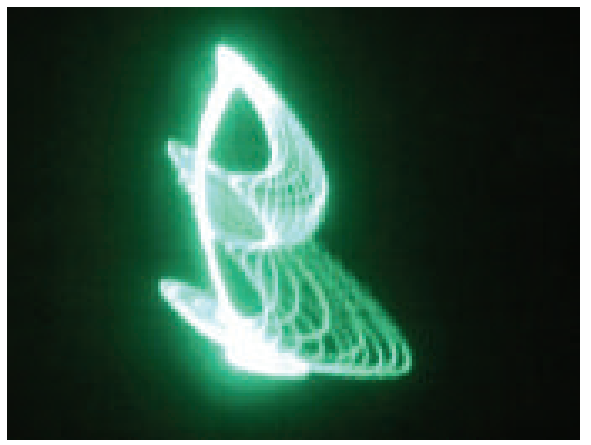

(e)

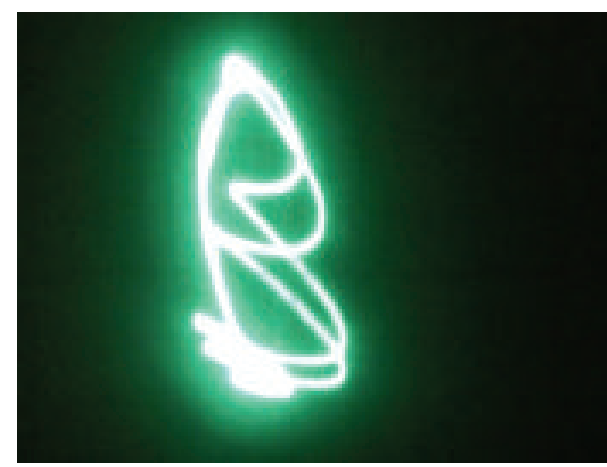

(b)

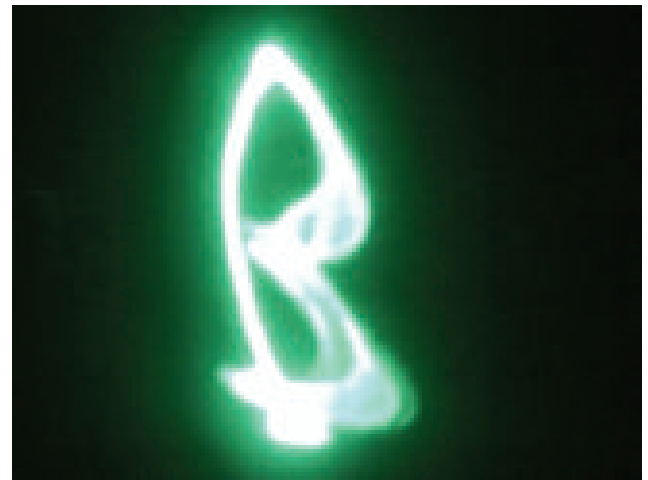

(d)

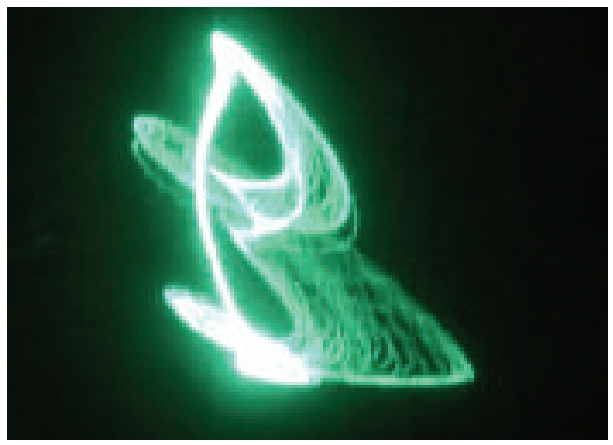

(f)

FIGURE 12: Experimental periodic and chaotic orbits on $i$ (volts/div. $=0.5)-\varphi$ (volts/div. $=0.1$ ) plane with (a) $\beta=0.4$, (b) $\beta=0.387$, (c) $\beta=0.37$, (d) $\beta=0.3883$, (e) $\beta=0.38$, and (f) $\beta=0.3772$.

For a binary sequence, which is generated by the proposed memristor oscillator and contains "0" and " 1 " of a given length $n$, this sequence is divided into $k$ nonoverlapping parts where each length is $m(k=n / m)$, where $n=1,000,000,000$ and $m=1000$.

An analysis report is generated by the test suite package, which consists of some relevant intermediate values, including proportions and $P$ values for each test. The analysis report is shown in Table 1. For comparison, the analysis report of the well-known Lorenz oscillator is shown in Table 2.

From Table 1 we can see that all of the statistical test's $P$ values pass the test. However, for the well-known Lorenz oscillator the tested item "Overlapping Template" fails to pass the test.
Moreover, from Tables 1 and 2, although both the memristor oscillator and the Lorenz oscillator all pass the tests of proportion, all the tested proportion items of memristor oscillator are greater than that for the Lorenz oscillator except for the item "Block Frequency."

Therefore, we can conclude that the random characteristic of the memristor oscillator is better than that of the wellknown Lorenz oscillator.

\section{Conclusion}

In conclusion, this paper has designed a nonautonomous flux-controlled memristor-based oscillator system by using the mathematical model of practical $\mathrm{HP} \mathrm{TiO}_{2}$ memristor. 


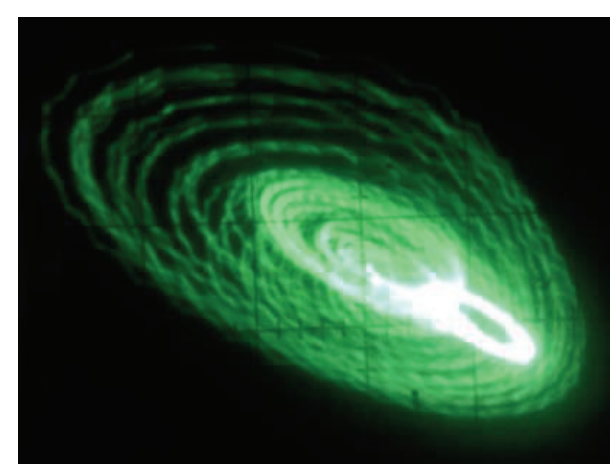

(a)

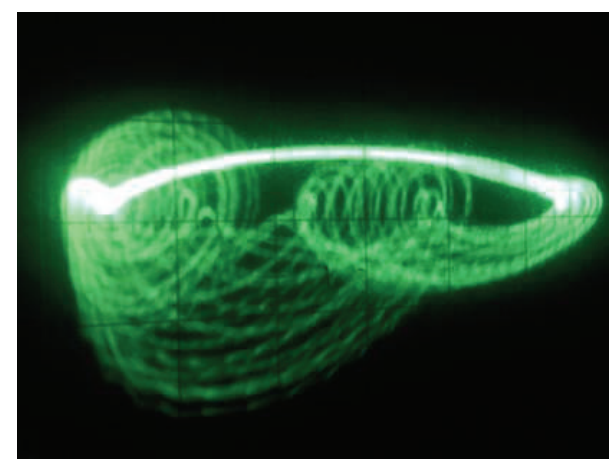

(c)

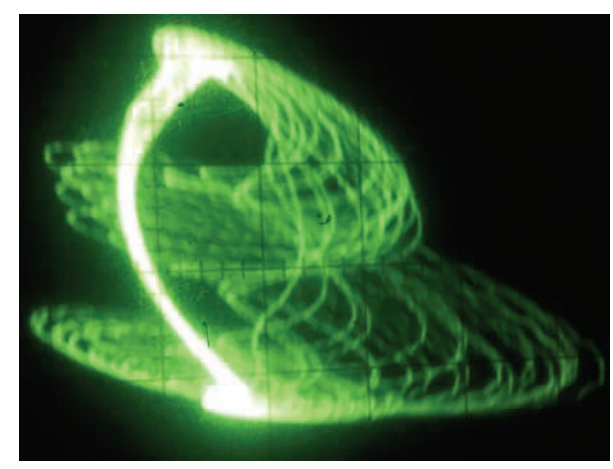

(b)

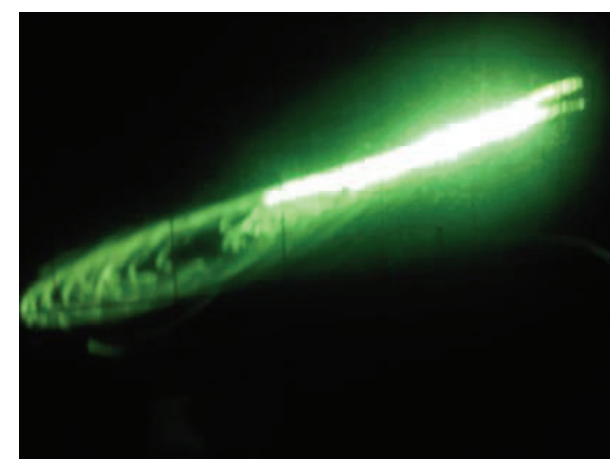

(d)

FIGURE 13: Experimental hyperchaotic orbits with $\beta=0.372$. (a) $v_{C}$ (volts/div. $\left.=0.5\right)-i$ (volts/div. $\left.=0.2\right)$ plane. $(\mathrm{b}) i($ volts $/$ div. $=1)-\varphi($ volts $/$ div. $=0.5)$ plane. $(\mathrm{c}) \varphi$ (volts $/$ div. $=0.2)-v_{C}($ volts $/$ div. $=0.5)$ plane. $(\mathrm{d}) v_{C}($ volts $/$ div. $=0.2)-i_{M}($ volts $/$ div. $=0.5)$ plane.

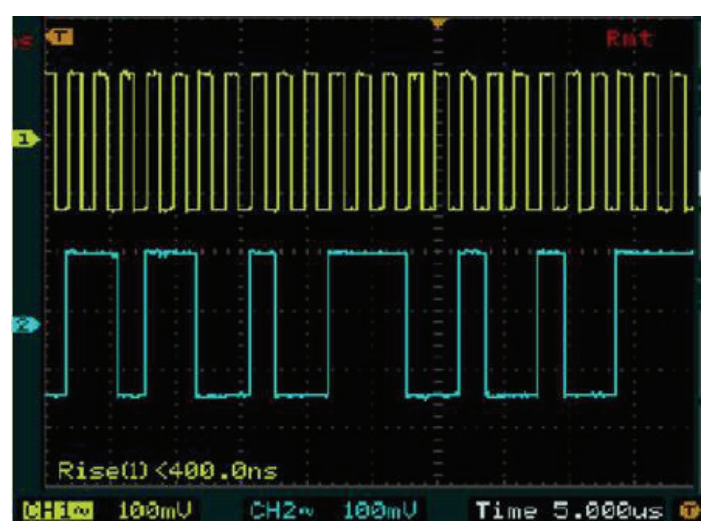

FIGURE 14: Experimental chaotic sequences generated by the proposed memristor-based oscillator circuit.

This proposed system possesses many specialties: (i) the system exhibits complex bifurcation from period to chaos and then to hyperchaos; (ii) when external excitation voltage is zero, this nonautonomous system degrades into autonomous system with a periodic oscillation; (iii) this system has some special phenomena, such as burst chaos, and irregular bifurcation started from period 3 but without period-doubling bifurcations; and (iv) it has no transient chaos or intermittence chaos which is an advantage for generating continuous chaotic sequences. In addition, DSP experiment shows that
TABLE 1: Depiction of the test report for memristor oscillator.

\begin{tabular}{lcc}
\hline Statistical test & $P$ value & Proportion \\
\hline Frequency & 0.781106 & 0.9900 \\
Block Frequency & 0.484646 & 0.9930 \\
Cumulative sums & 0.844641 & 0.9890 \\
Runs & 0.370262 & 0.9900 \\
Longest run & 0.562591 & 0.9930 \\
Rank & 0.262249 & 0.9900 \\
FFT & 0.452173 & 0.9870 \\
Nonoverlapping template & 0.601766 & 0.9940 \\
Overlapping Template & 0.837781 & 0.9850 \\
Universal & 0.674543 & 0.9920 \\
Approximate entropy & 0.809249 & 0.9900 \\
Random excursions & 0.867439 & 0.9937 \\
Random excursions variant & 0.984668 & 0.9937 \\
Serial & 0.550347 & 0.9950 \\
Linear complexity & 0.433590 & 0.9910 \\
\hline
\end{tabular}

DSP technology can be used to realize the chaotic memristorbased system and in realistic applications of information processing for generating $\mathrm{PN}$ sequences and performing various operations such as encryption and decryption. Actually, only using a mathematical model of the HP memristor rather than the actual memristor device, we can build an oscillator and realize a pseudorandom sequence generator based on 
TABLE 2: Depiction of the test report for Lorenz oscillator.

\begin{tabular}{lcc}
\hline Statistical test & $P$ value & Proportion \\
\hline Frequency & 0.387264 & 0.9870 \\
Block Frequency & 0.721777 & 0.9940 \\
Cumulative sums & 0.883171 & 0.9870 \\
Runs & 0.591409 & 0.9870 \\
Longest run & 0.428095 & 0.9910 \\
Rank & 0.829047 & 0.9860 \\
FFT & 0.027313 & 0.9850 \\
Nonoverlapping template & 0.452173 & 0.9840 \\
Overlapping Template & $0.003322^{*}$ & 0.9840 \\
Universal & 0.624627 & 0.9850 \\
Approximate entropy & 0.358641 & 0.9870 \\
Random excursions & 0.356948 & 0.9812 \\
Random excursions variant & 0.326626 & 0.9906 \\
Serial & 0.666245 & 0.9900 \\
Linear complexity & 0.801865 & 0.9850 \\
\hline
\end{tabular}

${ }^{*}$ is the tag that meaning didn't pass the test item.

DSP chip, which provides great convenience for designing and implementing a memristor-based oscillator in actual applications.

The statistical property of the memristor-based chaotic oscillator has been tested by using the NIST test suite. Tested results show that random characteristic of the oscillator is definitely up to the standards of the NIST and is better than that of the well-known Lorenz system. Therefore, this memristor-based oscillator can be used to design pseudorandom sequence generators for various applications of information safety filed.

\section{Conflict of Interests}

The authors declare that there is no conflict of interests regarding the publication of this paper.

\section{Acknowledgments}

This work was supported by the National Natural Science Foundation of China under Grant nos. 61271064, 61401134, and 60971046, the Natural Science Foundation of Zhejiang Province, China, under Grant nos. LZ12F01001, LQ14F010008, and LQ14F010009, and the Program for Zhejiang Leading Team of S\&T Innovation under Grant no. 2010R50010.

\section{References}

[1] L. O. Chua, "Memristor-the missing circuit element", IEEE Transactions on Circuit Theory, vol. 18, no. 5, pp. 507-519, 1971.

[2] D. B. Strukov, G. S. Snider, D. R. Stewart, and R. S. Williams, "The missing memristor found," Nature, vol. 453, pp. 80-83, 2008.

[3] M. Di Ventra, Y. V. Pershin, and L. O. Chua, "Circuit elements with memory: memristors, memcapacitors, and meminductors," Proceedings of the IEEE, vol. 97, no. 10, pp. 1717-1724, 2009.
[4] P. Junsangsri and F. Lombardi, "Design of a hybrid memory cell using memristance and ambipolarity," IEEE Transactions on Nanotechnology, vol. 12, no. 1, pp. 71-80, 2013.

[5] A. Thomas, "Memristor-based neural networks," Journal of Physics D: Applied Physics, vol. 46, no. 9, Article ID 093001, 2013.

[6] A. Buscarino, L. Fortuna, M. Frasca, and L. V. Gambuzza, "A chaotic circuit based on Hewlett-Packard memristor," Chaos, vol. 22, no. 2, Article ID 023136, 2012.

[7] S. Wen, Z. Zeng, and T. Huang, "Adaptive synchronization of memristor-based Chua's circuits," Physics Letters A, vol. 376, no. 44, pp. 2775-2780, 2012.

[8] X.-B. Tian and H. Xu, "Characteristics of titanium oxide memristor with coexistence of dopant drift and a tunnel barrier," Chinese Physics B, vol. 23, no. 6, Article ID 068401, 2014.

[9] L. Chua, "Memristor, hodgkin-huxley, and edge of chaos," Nanotechnology, vol. 24, no. 38, Article ID 383001, 2013.

[10] M. Itoh and L. O. Chua, "Memristor oscillators," International Journal of Bifurcation and Chaos, vol. 18, no. 11, pp. 3183-3206, 2008.

[11] H. Li, L. Wang, and S. Duan, "A memristor-based scroll chaotic system-design, analysis and circuit implementation," International Journal of Bifurcation and Chaos, vol. 24, no. 7, Article ID 1450099, 2014.

[12] B. Bao, F. Hu, Z. Liu, and J. Xu, "Mapping equivalent approach to analysis and realization of memristor-based dynamical circuit," Chinese Physics B, vol. 23, no. 7, Article ID 070503, 2014.

[13] Y. Lu, X. Huang, S. He, D. Wang, and B. Zhang, "Memristor based van der pol oscillation circuit," International Journal of Bifurcation and Chaos, vol. 24, no. 12, Article ID 1450154, 2014.

[14] B. Bao, P. Jiang, H. Wu, and F. Hu, "Complex transient dynamics in periodically forced memristive Chua's circuit," Nonlinear Dynamics, vol. 79, no. 4, pp. 2333-2343, 2015.

[15] G.-Y. Wang, J.-L. He, F. Yuan, and C.-J. Peng, "Dynamical behaviors of a $\mathrm{TiO}_{2}$ memristor oscillator," Chinese Physics Letters, vol. 30, no. 11, Article ID 110506, 2013.

[16] L. Wang, E. Drakakis, S. Duan, P. He, and X. Liao, "Memristor model and its application for chaos generation," International Journal of Bifurcation and Chaos, vol. 22, no. 8, Article ID 1250205, 2012. 


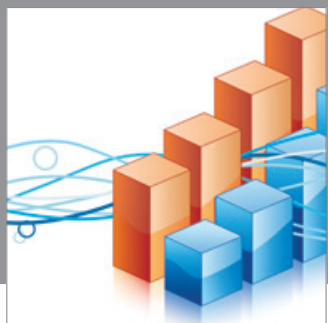

Advances in

Operations Research

mansans

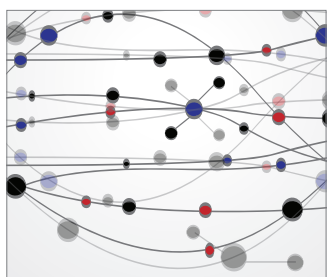

The Scientific World Journal
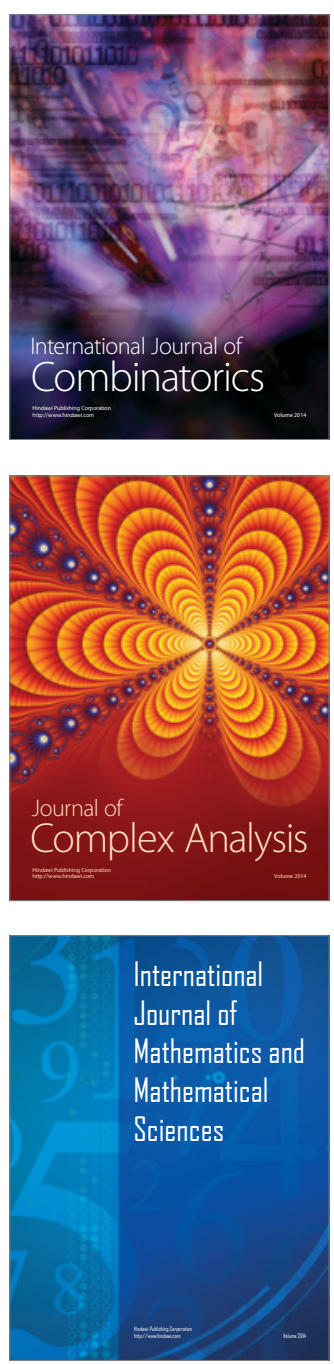
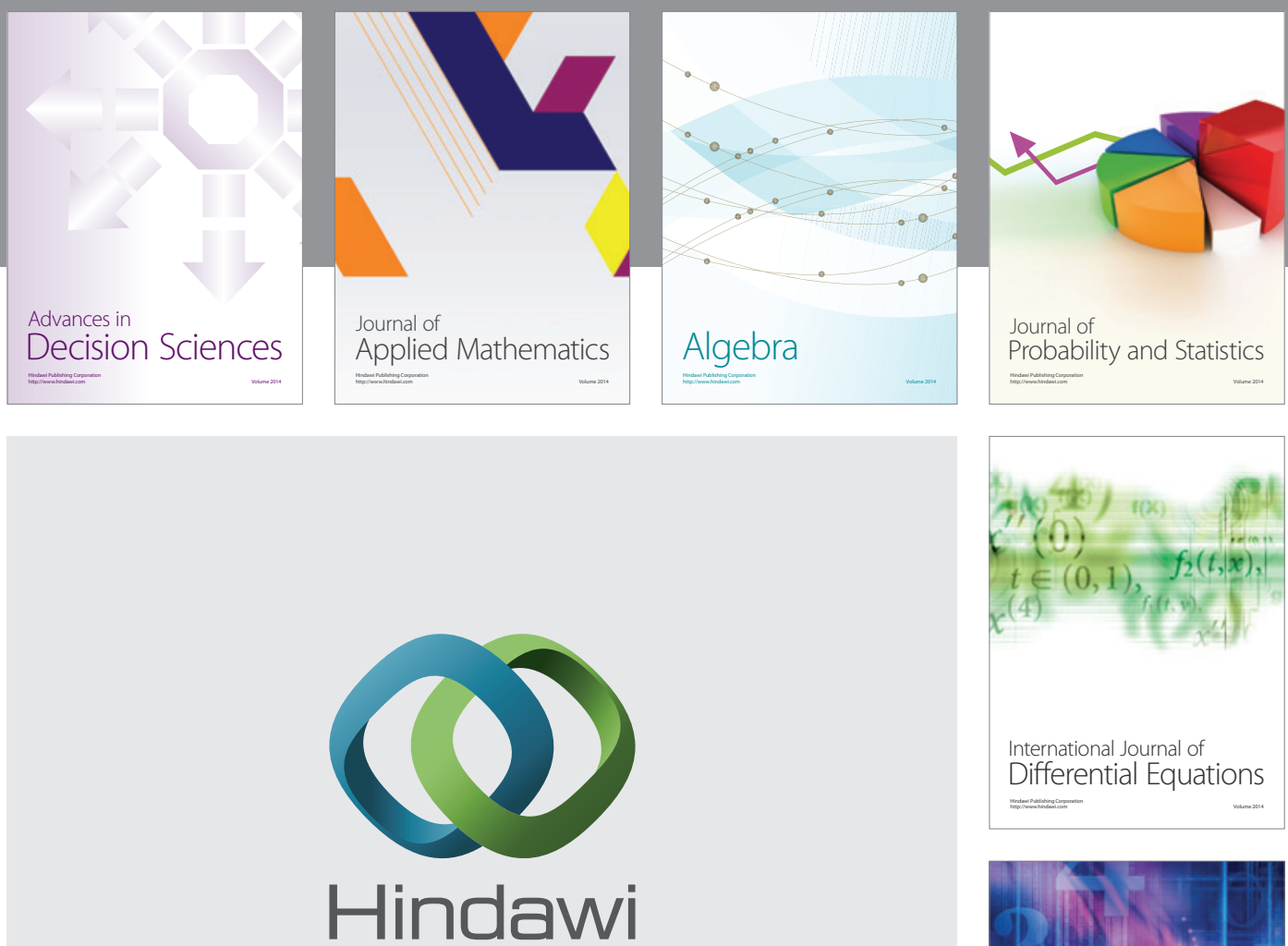

Submit your manuscripts at http://www.hindawi.com
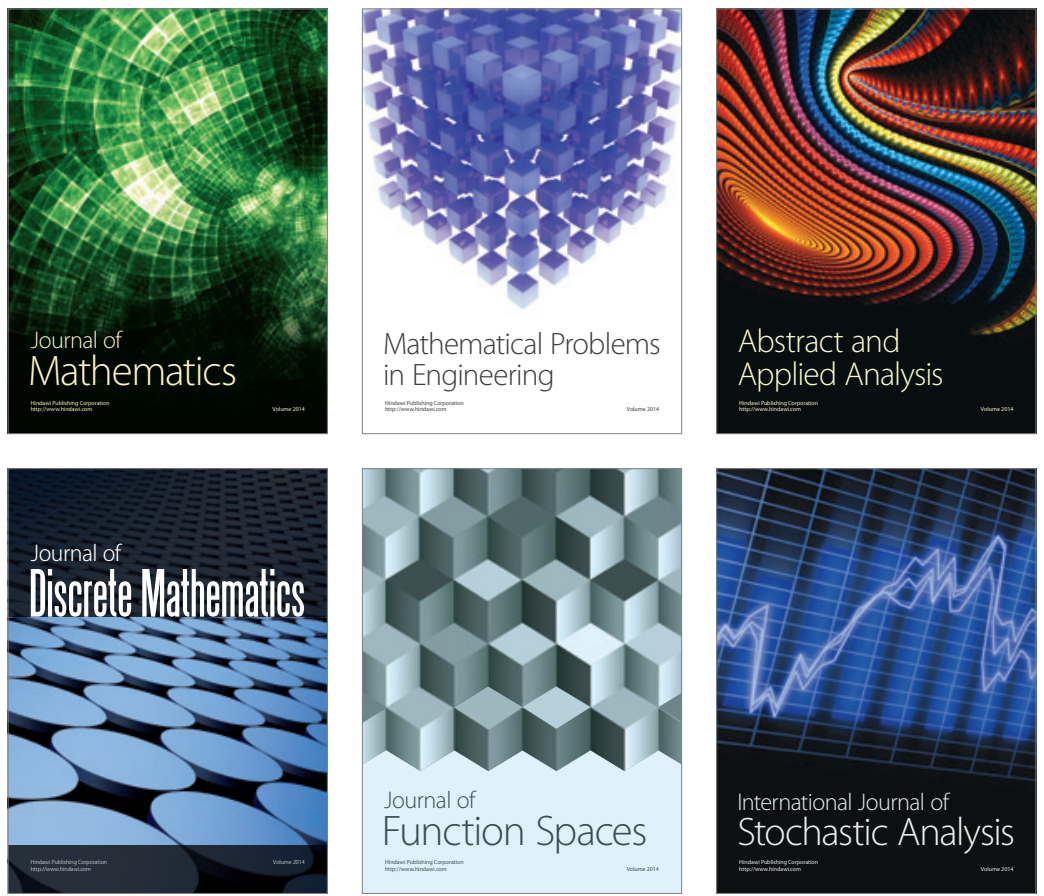

Journal of

Function Spaces

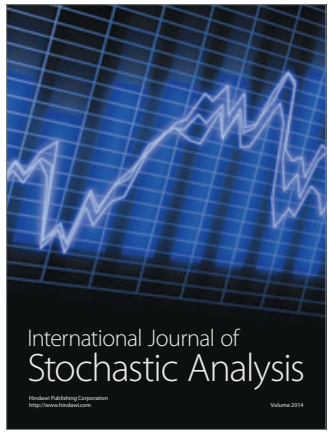

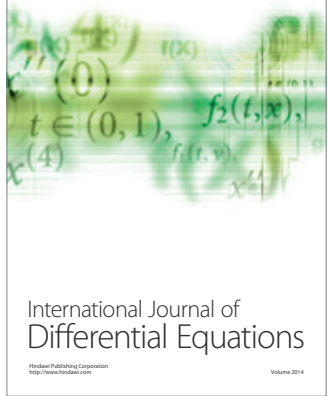
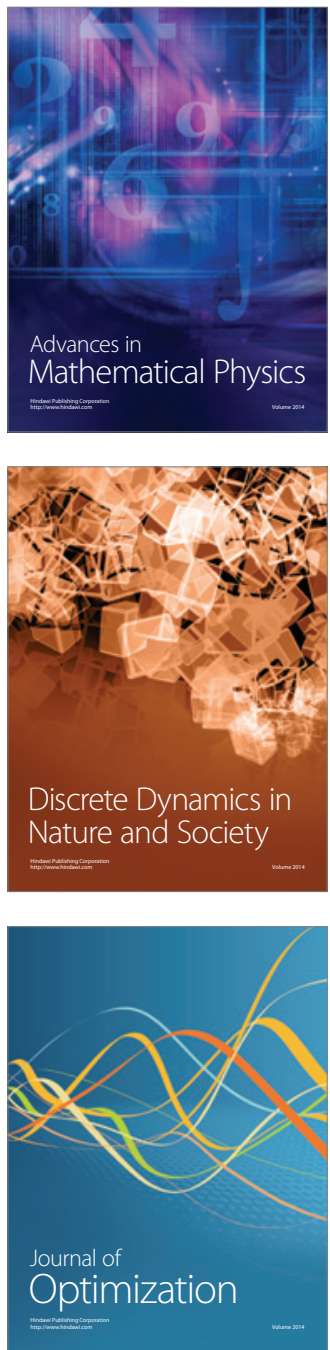